\title{
A prospective feasibility study to evaluate neoadjuvant-synchronous $S-1$ with radiotherapy for locally advanced rectal cancer: A multicentre phase II trial
}

\author{
MASAFUMI INOMATA ${ }^{1}$, TOMONORI AKAGI ${ }^{1}$, KENTARO NAKAJIMA ${ }^{1}$, TSUYOSHI ETOH $^{1}$, \\ KOICHIRO TAHARA ${ }^{2}$, TOSHIFUMI MATSUMOTO ${ }^{3}$, TADASHI OGAWA ${ }^{4}$, \\ KYUZO FUJII $^{5}$, AKIO SHIROMIZU ${ }^{6}$ and SEIGO KITANO ${ }^{1}$
}

\begin{abstract}
${ }^{1}$ Department of Gastroenterological and Pediatric Surgery, Oita University Faculty of Medicine, Oita 879-5593;
${ }^{2}$ Department of Surgery, National Hospital Organization, Oita Medical Center, Oita 879-5593; ${ }^{3}$ Department of Gastroenterological Surgery, National Hospital Organization Beppu Medical Center, Beppu 874-0011; ${ }^{4}$ Department of Surgery, Oita Prefectural Hospital, Bunyo 870-8511; ${ }^{5}$ Department of Surgery, Nakatsu Municipal Hospital, Nakatsu 871-8511; ${ }^{6}$ Department of Surgery, Oita Red Cross Hospital, Oita 870-0033, Japan
\end{abstract}

Received June 29, 2015; Accepted January 13, 2016

DOI: $10.3892 / \mathrm{mco} .2016 .767$

\begin{abstract}
Treatment results of locally advanced rectal cancer without preoperative chemoradiotherapy (CRT) in Japan do not differ from those of Western countries. Preoperative CRT with new anticancer agents may decrease local recurrence rate and prevent distant metastases, thus improving survival. We conducted a trial to evaluate feasibility of neoadjuvant CRT using S-1 in patients with locally advanced rectal cancer. A multi-institutional (17 specialized centres), interventional, phase II trial was conducted from April 2009 to August 2011. Patients fulfilling the following requirements before neoadjuvant CRT were included: histologically proven rectal carcinoma; tumour in the upper or lower rectum; cancer classified as T3-4 N0-3 M0. Neoadjuvant CRT with S-1 $\left(80 \mathrm{mg} / \mathrm{m}^{2} /\right.$ day on days $1-5,8-12,22-26$, and 29-33) and irradiation (total 45 Gy/25 fr, 1.8 Gy/day, on days 1-5, 8-12, 15-19, 22-26, and 29-33) was performed. Total mesorectal excision with D3 lymphadenectomy was performed during weeks 4 and 8 after completion of neoadjuvant CRT. The primary endpoint was completion rate of neoadjuvant CRT. Secondary endpoints were response rate to neoadjuvant CRT, short-term clinical outcomes, curative resection rate, and pathologic response (grade 2/3). Of the 37 patients included, $86.5 \%$ completed neoadjuvant CRT (95\% CI, 75.5-97.5\%), and $10.8 \%$ (4) experienced an adverse event (grade 3/4). Response rate (RECIST 1.0) was $56.8 \%$ (95\% CI, 40.8-72.7\%), and pathologic response rate
\end{abstract}

Correspondence to: Professor Masafumi Inomata, Department of Gastroenterological and Pediatric Surgery, Oita University Faculty of Medicine, 1-1 Idaigaoka, Hasama-machi, Oita 879-5593, Japan

E-mail: inomata@oita-u.ac.jp

Key words: neoadjuvant, S-1+radiotherapy, rectal cancer, multicentre phase II trial was $48.6 \%$ (95\% CI, 32.5-64.8\%). This study demonstrated that neoadjuvant-synchronous S-1+radiotherapy for locally advanced rectal cancer was feasible in terms of pathologic response and adverse events. Registration number: UMIN-CTR, No. C003396.

\section{Introduction}

Neoadjuvant therapy is proven to be effective in reducing the risk of local recurrence of cancer in populations in Western countries, and it is included in the standard therapy for local, advanced rectal cancer (1). Compared with surgery alone, local neoadjuvant therapy improves disease control; however, a survival benefit of this therapy has been reported in only one study (2). Even in the era of total mesorectal excision, the local control benefit of preoperative radiotherapy remains relevant (3). The feasibility of the addition of chemotherapy to preoperative conventional long-term radiotherapy, along with its enhanced tumouricidal effects, has been shown (4). In Japan, total mesorectal excision or tumour-specific mesorectal excision followed by adjuvant chemotherapy without preoperative treatment is a standard treatment strategy, and lateral lymph node dissection is provided as an additional treatment in patients with lower rectal cancer (5). Surgical treatment without radiotherapy in Japan has shown results that are no worse than those in Western countries using chemoradiotherapy (CRT) with surgery, and extrapolating the results of preoperative treatment in Western countries to Japan would be controversial. The Japanese Classification of Colorectal Carcinoma (6) describes that the standard treatment is surgery without radiotherapy, based on several reports, which demonstrated a low 5-year local recurrence rate of 3.0-6.9\% (7-9).

Although previously published CRT studies have similar techniques and doses of irradiation, the administration of chemotherapy varies markedly $(10,11)$. The use of new anticancer agents may better control locally advanced rectal cancer, and several clinical trials of neoadjuvant CRT have focused on 
determining the optimal chemotherapy component. In Western countries, novel treatment strategies have been tested, including the addition of new cytotoxic drugs and/or molecular-targeted drugs to fluoropyrimidine-based CRT, either concurrently or prior to CRT $(12,13)$. However, since oral treatment can be administered on an outpatient basis and thus reduces the length of the hospital stay, oral chemotherapy appears to have some advantages over intravenously administered treatment, in terms of pharmacoeconomics and patient preference (14). In the future, the role of oral chemotherapy in treating malignant disease is expected to increase significantly. S-1 (TS-1, Taiho Pharmaceutical) is an orally active combination of tegafur [a prodrug of fluorouracil (5-FU)], gimeracil (an inhibitor of dihydropyrimidine dehydrogenase, which degrades fluorouracil), and oteracil potassium (which inhibits the phosphorylation of fluorouracil in the gastrointestinal tract, thereby reducing the gastrointestinal toxic effects of fluorouracil) in a molar ratio of 1:0.4:1 $(15,16)$. Two phase II trials involving patients with advanced or recurrent colorectal cancer reported that the response rate to treatment with S-1 alone exceeded $40 \%(17,18)$. Furthermore, $\mathrm{S}-1$ enhances the radiation response of human colon cancer xenografts that are resistant to 5-FU (19). Therefore, the present, phase II study was conducted to confirm the feasibility of CRT with S-1 before surgery as a therapeutic strategy for advanced rectal cancer.

\section{Patients and methods}

Protocol of the OITA TRIAL. The study design of the prospective, multi-institutional study entitled the OITA TRIAL 1 has been published previously (20). The protocol of the present study was approved by the Oita University Clinical Trial Review Committee and the institutional review board of each participating hospital, and all patients provided written, informed consent. The present study was registered at the UMIN Clinical Trial Registry as UMIN000003396 (further details accessible at: http://www.umin.ac.jp/ctr/index.htm).

Purpose. The purpose of the current study was to evaluate the feasibility of neoadjuvant CRT with S-1 for locally advanced rectal cancer.

Study setting. The present multi-institutional, interventional, phase II trial was conducted in 17 specialized centres within Japan.

Endpoints. The primary endpoint of the present study was the rate of treatment completion of neoadjuvant CRT. Treatment completion was defined as the patient receiving an administered dose of radiotherapy and S-1 of $>75 \%$ of the planned dose. Secondary endpoints were the response rate of neoadjuvant CRT, short-term clinical outcomes, rate of curative resection, and pathologic evaluation. The short-term clinical outcomes included adverse events of neoadjuvant CRT and surgery-related complications. Response rate was evaluated using the Response Evaluation Criteria in Solid Tumors (RECIST) v1.1 (21), and adverse events including those of preoperative CRT and surgical complications were evaluated using Common Terminology Criteria for Adverse Events (CTCAE) v4.0, available at: http://ctep.cancer.gov.
Eligibility criteria. Tumours were staged according to the tumor-node-metastasis (TNM) classification system (Union for International Cancer Control, UICC 6th edition) (20).

Inclusion and exclusion criteria. For inclusion in the present study, patients had to fulfill the following requirements before enrolment: i) Histologically proven rectal carcinoma; ii) tumour located in the rectum ( $\mathrm{Ra}, \mathrm{Rb}, \mathrm{P})$; iii) cancer classified as T3-4 N0-3 M0, according to the TNM classification system (17); iv) no bowel obstruction; v) age $>20$ and $<80$ years; vi) sufficient organ function; vii) no previous history of gastrointestinal surgery; viii) no previous history of chemotherapy or radiotherapy; and ix) the patient has provided written informed consent.

The exclusion criteria for the present study included: i) Synchronous or metachronous (within 5 years) malignancy, other than carcinoma in situ; ii) critical drug sensitivity to S-1; iii) severe pulmonary emphysema, interstitial pneumonitis, or ischaemic heart disease; iv) pregnant or lactating women; v) severe mental disease; and vi) the patient is undergoing continuous systemic steroid therapy.

Treatment method. S-1 was administered orally, twice daily on days 1-5, 8-12, 22-26, and 29-33. The dose of S-1 was assigned according to patient body surface area (BSA): Patients with a BSA of less than $1.25 \mathrm{~m}^{2}$ received a dose of $80 \mathrm{mg} /$ day, those with a BSA of between $1.25 \mathrm{~m}^{2}$ and $<1.5 \mathrm{~m}^{2}$ received a dose of $100 \mathrm{mg} /$ day, and those with a $\mathrm{BSA}$ of $>1.5 \mathrm{~m}^{2}$ received a dose of $120 \mathrm{mg} /$ day. Radiotherapy was performed on days $1-5$, 8-12, 15-19, 22-26, and 29-33 with 1.8 Gy/day (total dose of $45 \mathrm{~Gy} / 25 \mathrm{fr})$.

Additional treatment. Resection of the rectum with D3 lymphadenectomy was performed according to the Japanese Classification of Colorectal Carcinoma (Japanese Society for Cancer of the Colon and Rectum) and General Rules for Clinical and Pathological Studies on Cancer of the Colon, Rectum and Anus, 6th ed, 1998 (in Japanese). Surgery was performed during the 4th and 8th weeks following the end of the neoadjuvant CRT. Proposed operations were anterior resection with or without covering ileostomy and anterior peritoneal resection. In cases where the preoperative and intraoperative findings indicated that lateral lymph node metastasis was not suspected, lateral lymph node dissection was not performed. Adjuvant chemotherapy was not specified.

Follow-up. Patients were observed by their surgeon every 3-4 months following the operation. Blood tests, abdominal computed tomography, and plain chest X-ray were performed at each visit.

Study design and statistical methods. The present study was designed to assess the feasibility of neoadjuvant CRT with S-1 for locally advanced rectal cancer in terms of treatment completion rate, efficacy, adverse events of neoadjuvant CRT, and curative resection ( $\mathrm{R} 0$ resection) rate. If the feasibility and efficacy of neoadjuvant CRT with S-1 were shown, then neoadjuvant CRT with S-1 was determined to be the preferred treatment. The planned sample size was 35 patients, which was calculated by the Southwest Oncology Group's method $(18,19)$, 
Table I. Baseline patient characteristics.

\begin{tabular}{lc}
\hline Characteristic & $\begin{array}{c}\text { No. of patients }(\%) \\
\mathrm{n}=37\end{array}$ \\
\hline Age (years) & 59 \\
Median & $32-79$ \\
Range & \\
Gender & $23(62.2)$ \\
Male & $24(37.8)$ \\
Female & \\
Tumor location & $9(24.4)$ \\
Ra & $27(73.0)$ \\
Rb & $1(2.8)$ \\
P & \\
Clinical TNM stage & \\
T3 & $32(86.5)$ \\
T4 & $5(13.5)$ \\
N0 & $10(27.0)$ \\
N1 & $18(48.6)$ \\
N2 & $8(21.6)$ \\
N3 & $1(2.8)$ \\
\hline
\end{tabular}

TNM, tumour-node-metastasis; Ra, upper rectum; Rb, lower rectum; $\mathrm{P}$, anorectum.

Table II. Acute adverse events associated with preoperative chemoradiotherapy.

No. of patients $(n=37)$

\begin{tabular}{lcc}
\cline { 2 - 3 } Adverse event & Grade 3 & Grade 4 \\
\hline Diarrhoea & 2 & 0 \\
Anaemia & 1 & 0 \\
Leukopaenia & 1 & 0 \\
Thrombocytopaenia & 1 & 0 \\
\hline
\end{tabular}

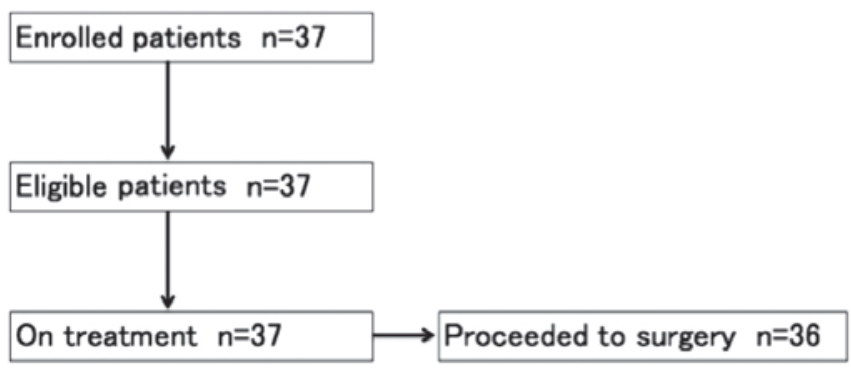

Figure 1. Study diagram of the 37 patients enrolled in the current study.

based on an assumed rate of treatment completion of $90 \%$ and a threshold rate of $70 \%$, with a one-sided alpha error of 0.05 and power of $90 \%$. Allowing for a $5 \%$ loss to follow-up, the method calculated that 35 patients were required.

\section{Results}

Thirty-seven patients were enrolled at the 17 participating hospitals between April 2009 and October 2011. All 37 patients were evaluated according to the intention-to-treat principle. A flow diagram of the 37 enrolled patients is shown in Fig. 1, and patient demographics and tumour characteristics are listed in Table I.

Treatment. Treatment completion with neoadjuvant CRT was attained by $32(86.5 \%)$ of the 37 patients $(95 \%$ CI, 75.5-97.5\%). The rate of treatment completion was $94.6 \%$ for the radiotherapy group and $89.2 \%$ for the S-1 group. Only one patient did not undergo surgery because multiple liver metastases developed that were incurable after complete neoadjuvant CRT. Of the 36 patients undergoing surgery, 18 (48.6\%) underwent abdominoperineal resection, $13(35.1 \%)$ underwent anterior resection, $3(8.1 \%)$ underwent intersphincteric resection, and Hatmann's operation was necessary in $2(5.4 \%)$ patients.

Safety. Most adverse events were mild to moderate in nature. Grade 3 adverse events, which occurred in 4 (10.8\%) patients, are listed in Table II. The 5 events occurring in the 4 patients were judged to be treatment related. No patient experienced any grade 4 adverse event. The most common adverse event was grade 1-2 diarrhoea and anorexia, which was manageable (data not shown).

Clinical response rate, pathologic evaluation, and rate of curative resection. The clinical response rate, pathological evaluation, and rate of curative resection are shown in Table III. The response rate (RECIST 1.1) was 56.8\% (95\% CI; 40.8-72.7\%), and the pathologic response rate (grade $2 / 3$ ) was $48.6 \%$ (95\% CI; 32.5-64.8\%). The pathologic response rate in all of the eligible patients, defined by a degeneration/necrosis area $>1 / 3$ (Grades 1b, 2, and 3), was $75.7 \%$ (28/37). Of the 36 patients who underwent surgery, $\mathrm{R} 0$ resection was performed in 35 and $\mathrm{R} 1$ in one patient due to a positive circumferential resection margin. The proportion of $\mathrm{R} 0$ resections performed in all 37 eligible patients was $94.6 \%$ (95\% CI, 87.3-99.3\%).

\section{Discussion}

In Western countries, patients with advanced rectal cancer are routinely treated with preoperative radiotherapy together with anti-cancer agents, whereas the standard treatment in Japan is adjuvant chemotherapy followed by surgery. We believe that survival may be improved by preoperative treatment with the new anticancer agent $\mathrm{S}-1$ as it is expected to decrease local recurrence due to its effect of bulk reduction, obtain a high rate of complete treatment of neoadjuvant CRT, and prevent distant metastases. Therefore, the present multicentre phase II study was conducted to evaluate the feasibility of neoadjuvant CRT using S-1 in patients with locally advanced rectal cancer. The administration schedule of S-1 was $80 \mathrm{mg} / \mathrm{m}^{2} /$ day for 5 days, and no treatment for 2 days, resulting in a total weekly dose of S-1 of $400 \mathrm{mg} / \mathrm{m}^{2} /$ week. By contrast, S-1 administered at $80 \mathrm{mg} / \mathrm{m}^{2} /$ day for 7 days is the standard dose when used as a single agent for systemic therapy, which totals $560 \mathrm{mg} / \mathrm{m}^{2} /$ week. As the total dose of S-1 per week in the present study $\left(400 \mathrm{mg} / \mathrm{m}^{2}\right)$ is less 
Table III. Response rate and pathological findings.

Finding

No. patients $(n=37)$

\begin{tabular}{lr}
\hline Downstaging & 2 \\
Complete response & 19 \\
Partial response & 15 \\
Stable disease & 1 \\
Progressive disease & \\
Curability & 35 \\
R0 & 1 \\
R1 & 0 \\
R2 & \\
Pathological TNM stage & 3 \\
T1 & 5 \\
T2 & 25 \\
T3 & 3 \\
T4 & 24 \\
N0 & 7 \\
N1 & 2 \\
N2 & 2 \\
N3 & 14 \\
GCCR pathological response & 4 \\
Grade 1a & 10 \\
Grade 1b & 1 \\
Grade 2 & \\
Grade 3 & 1 \\
\hline
\end{tabular}

JSCCR, Japanese Society for Cancer of the Colon and Rectum; TNM, tumour-node-metastasis classification (UICC); R0, no residual tumor; R1, positive surgical margin; R2, macroscopic residual tumor.

than the standard amount per week $\left(560 \mathrm{mg} / \mathrm{m}^{2}\right)$, a phase I trial was not conducted. The current study identified that treatment with neoadjuvant-synchronous S-1 + radiotherapy for locally advanced rectal cancer was feasible in terms of pathological response and adverse events. The data of the present phase II study can be compared with those of previous major phase III clinical trials in Table III $(12,22-27)$. Almost all of the patients completed their full course of chemotherapy and radiotherapy. The treatment results were comparable with those of similar studies and exhibited high compliance for preoperative CRT.

The tolerability of CRT using S-1 was acceptable in the present study. However, whether orally active fluoropyrimidines can replace fluorouracil/leucovorin calcium (5-FU/LV) delivered by infusion (plus oxaliplatin) during CRT remains unanswered. The present study revealed the compliances for chemotherapy and radiotherapy were $89.2 \%$ and $94.6 \%$, respectively. Poor compliance indicated that the chemotherapy regimen was too toxic, and so treatment was discontinued. We considered there to be two reasons for the high compliance rates: Oral treatment could be administered on an outpatient basis, and S-1 was less toxic than intravenously delivered 5-FU/LV (plus oxaliplatin).

The efficacy and tolerability of the S-1-based CRT reported herein are comparable with those previously reported for other fluoropyrimidines, and are improved compared to those reported with cytotoxic drug combinations. In 2011, Sadahiro et al (28) reported that the efficacy of CRT with S-1 appears to be equivalent to the efficacy reported for CRT with capecitabine. However, the schedule of S-1 in our study is different from that used by Sadahiro et al. The present study was planned in order to obtain increased feasibility. Although there were several differences between examination items and standards, the present study demonstrates almost equivalent results in terms of high preoperative treatment compliance and R0 resection rate and a lower rate of Grade 3/4 adverse events (10.8\% vs. $22.2 \%$ ) compared with that of Sadahiro et al, which used an S-1 regimen (28). However, the pathologic complete response rate of $10.8 \%$ with our regimen was lower than the $22.2 \%$ rate of the Sadahiro et al study (28). Nevertheless, we believe that the $\mathrm{S}-1$ regimen in the preoperative CRT setting is a key drug regimen and is worthy of further examination.

Investigations into various genetic and molecular biomarkers that may allow prediction of the response to preoperative CRT have been reported previously (29,30). To date, an association with the therapeutic response to preoperative CRT has been reported for p53, (31-34) epidermal growth factor receptor, (35-37) Ki-67, (38,39) Bcl-2, (40) and Bax (41). Due to controversial and inconclusive results, however, none of these specific molecular markers have been definitively proven to be predictors of a response to CRT. Thus, to identify patients who might derive clinical benefit from preoperative CRT, improved predictive tools are necessary. We are attempting to detect useful molecular biomarkers using the biopsy samples obtained from the patients in the present study prior to the commencement of the preoperative CRT.

The present study demonstrates the feasibility, efficacy and tolerability of CRT using S-1. A limitation of this study is its small sample size, and further investigations are therefore necessary. In conclusion, preoperative CRT with S-1 was a well-tolerated treatment regimen that achieved an excellent $\mathrm{R} 0$ resection rate in $94.6 \%$ of patients with locally advanced rectal cancer. Further trials are required to confirm the benefits of adding S-1 in preoperative CRT for rectal cancer.

\section{Acknowledgements}

The present study was supported in part by a grant-in-aid for Clinical Cancer Research from the Japanese Ministry of Health, Labour and Welfare (grant no. 22-Clinical Cancer-027).

\section{References}

1. Willett CG, Czito BG and Bendell JC: Radiation therapy in stage II and III rectal cancer. Clin Cancer Res 13: 6903s-6908s, 2007.

2. Improved survival with preoperative radiotherapy in resectable rectal cancer. Swedish rectal cancer trial. N Engl J Med 336: 980-987, 1997.

3. Kapiteijn E, Marijnen CA, Nagtegaal ID, Putter H, Steup WH, Wiggers T, Rutten HJ, Pahlman L, Glimelius B, van Krieken JH, et al: Preoperative radiotherapy combined with total mesorectal excision for resectable rectal cancer. N Engl J Med 345: 638-646, 2001.

4. Ceelen W, Fierens K, Van Nieuwenhove Y and Pattyn P: Preoperative chemoradiation versus radiation alone for stage II and III resectable rectal cancer: A systematic review and meta-analysis. Int J Cancer 124: 2966-2972, 2009.

5. Sugihara K, Kobayashi H, Kato T, Mori T, Mochizuki H, Kameoka S, Shirouzu K and Muto T: Indication and benefit of pelvic sidewall dissection for rectal cancer. Dis Colon Rectum 49: 1663-1672, 2006. 
6. Watanabe T, Itabashi M, Shimada Y, et al: Japanese Society for Cancer of the Colon and Rectum (JSCCR) Guideline 2014 for treatment of colorectal cancer. Int J Clin Oncol 20: 207-239, 2015.

7. Fukunaga Y, Higashino M, Tanimura S, Takemura M and Fujiwara Y: Laparoscopic rectal surgery for middle and lower rectal cancer. Surg Endosc 24: 145-151, 2010.

8. Fujita S, Yamamoto S, Akasu T and Moriya Y: Outcome of patients with clinical stage II or III rectal cancer treated without adjuvant radiotherapy. Int J Colorectal Dis 23: 1073-1079, 2008.

9. Kusters M, Beets GL, van de Velde CJ, Beets-Tan RG, Marijnen CA, Rutten HJ, Putter H and Moriya Y: A comparison between the treatment of low rectal cancer in Japan and the Netherlands, focusing on the patterns of local recurrence. Ann Surg 249: 229-235, 2009

10. Gérard JP, Conroy T, Bonnetain F, Bouché O, Chapet O, Closon-Dejardin MT, Untereiner M, Leduc B, Francois E, Maurel J, et al: Preoperative radiotherapy with or without concurrent fluorouracil and leucovorin in T3-4 rectal cancers: Results of FFCD 9203. J Clin Oncol 24: 4620-4625, 2006.

11. Bosset JF, Calais G, Mineur L, Maingon P, Radosevic-Jelic L, Daban A, Bardet E, Beny A, Briffaux A and Collette L: Enhanced tumorocidal effect of chemotherapy with preoperative radiotherapy for rectal cancer: Preliminary results-EORTC 22921. J Clin Oncol 23: 5620-5627, 2005.

12. Gérard JP, Azria D, Gourgou-Bourgade S, Martel-Laffay I, Hennequin C, Etienne PL, Vendrely V, François E, de La Roche G, Bouché O, et al: Comparison of two neoadjuvant chemoradiotherapy regimens for locally advanced rectal cancer: Results of the phase III trial ACCORD 12/0405-Prodige 2. J Clin Oncol 28: 1638-1644, 2010

13. Chua YJ, Barbachano Y, Cunningham D, Oates JR, Brown G, Wotherspoon A, Tait D, Massey A, Tebbutt NC and Chau I: Neoadjuvant capecitabine and oxaliplatin before chemoradiotherapy and total mesorectal excision in MRI-defined poor-risk rectal cancer: A phase 2 trial. Lancet Oncol 11: 241-248, 2010.

14. DeMario MD and Ratain MJ: Oral chemotherapy: Rationale and future directions. J Clin Oncol 16: 2557-2567, 1998.

15. Diasio RB: Clinical implications of dihydropyrimidine dehydrogenase inhibition. Oncology (Williston Park) 13 (7 Suppl 3) S17-S21, 1999

16. Shirasaka T, Shimamato Y, Ohshimo H, Yamaguchi M, Kato T, Yonekura K and Fukushima M: Development of a novel form of an oral 5-fluorouracil derivative (S-1) directed to the potentiation of the tumor selective cytotoxicity of 5-fluorouracil by two biochemical modulators. Anticancer Drugs 7: 548-557, 1996.

17. Shirao K, Ohtsu A, Takada H, Mitachi Y, Hirakawa K, Horikoshi N, Okamura T, Hirata K, Saitoh S, Isomoto H and Satoh A: Phase II study of oral S-1 for treatment of metastatic colorectal carcinoma. Cancer 100: 2355-2361, 2004.

18. Ohtsu A, Baba H, Sakata Y, Mitachi Y, Horikoshi N, Sugimachi K and Taguchi T: Phase II study of S-1, a novel oral fluorophyrimidine derivative, in patients with metastatic colorectal carcinoma. S-1 cooperative colorectal carcinoma study group. $\mathrm{Br}$ J Cancer 83: 141-145, 2000.

19. Nakata E, Fukushima M, Takai Y, Nemoto K, Ogawa Y, Nomiya T, Nakamura Y, Milas L and Yamada S: S-1, an oral fluoropyrimidine, enhances radiation response of DLD-1/FU human colon cance xenografts resistant to 5-FU. Oncol Rep 16: 465-471, 2006.

20. Inomata M, Akagi T, Nakajima K, Etoh T, Shiraishi N, Tahara K, Matsumoto T, Kinoshita T, Fujii K, Shiromizu A, et al: Prospective feasibility study to evaluate neoadjuvant-synchronous S-1+RT for locally advanced rectal cancer: A multicenter phase II trial (UMIN ID: 03396). Jpn J Clin Oncol 43: 321-323, 2013.

21. Eisenhauer EA, Therasse P, Bogaerts J, Schwartz LH, Sargent D, Ford R, Dancey, J, Arbuck S, Gwyther S, Mooney M, et al: New response evaluation criteria in solid tumours: Revised RECIST guideline (version 1.1). Eur J Cancer 45: 228-47, 2009.

22. Cassidy J, Clarke S, Díaz-Rubio E, Scheithauer W, Figer A, Wong R, Koski S, Lichinitser M, Yang TS, Rivera F, et al Randomized phase III study of capecitabine plus oxaliplatin compared with fluorouracil/folinic acid plus oxaliplatin as first-line therapy for metastatic colorectal cancer. J Clin Oncol 26: 2006-2012,2008.

23. Berry G, Smith CL, Macaskill P and Irwig L: Analytic methods for comparing two dichotomous screening or diagnostic tests applied to two populations of differing disease prevalence when individuals negative on both tests are unverified. Stat Med 21: 853-862, 2002

24. Green SJ and Dahlberg S: Planned versus attained design in phase II clinical trials. Stat Med 11: 853-862, 1992.
25. Aschele C, Cionini L, Lonardi S, Pinto C, Cordio S, Rosati G, Artale S, Tagliagambe A, Ambrosini G, Rosetti P, et al: Primary tumor response to preoperative chemoradiation with or without oxaliplatin in locally advanced rectal cancer: Pathologic results of the STAR-01 randomized phase III trial. J Clin Oncol 29: 2773-2780, 2011.

26. Gérard JP, Azria D, Gourgou-Bourgade S, Martel-Lafay I, Hennequin C, Etienne PL, Vendrely V, François E, de La Roche G, Bouché $\mathrm{O}$, et al: Clinical outcome of the ACCORD 12/0405 PRODIGE 2 randomized trial in rectal cancer. J Clin Oncol 30: 4558-4565, 2012.

27. Rödel C, Liersch T, Becker H, Fietkau R, Hohenberger W, Hothorn T, Graeven U, Arnold D, Lang-Welzenbach M, Raab HR, et al: Preoperative chemoradiotherapy and postoperative chemotherapy with fluorouracil and oxaliplatin versus fluorouracil alone in locally advanced rectal cancer: Initial results of the German CAO/ARO/AIO-04 randomised phase 3 trial. Lancet Oncol 13: 679-687, 2012.

28. Sadahiro S, Suzuki T, Tanaka A, Okada K, Kamijo A, Murayama C, Akiba T and Nakayama Y: Phase I/II study of preoperative concurrent chemoradiotherapy with S-1 for locally advanced, resectable rectal adenocarcinoma. Oncology 81: 306-311, 2011.

29. Kuremsky JG, Tepper JE and McLeod HL: Biomarkers for response to neoadjuvant chemoradiation for rectal cancer. Int $\mathrm{J}$ Radiat Oncol Biol Phys 74: 673-688, 2009.

30. Miura K, Fujibuchi W, Ishida K, Naitoh T, Ogawa H, Ando T, Yazaki N, Watanabe K, Haneda S, Shibata C and Sasaki I: Inhibitor of apoptosis protein family as diagnostic markers and therapeutic targets of colorectal cancer. Surg Today 41: 175-182, 2011.

31. Luna-Perez P, Arriola EL, Cuadra Y, Alvarado I and Quintero A: p53 protein overexpression and response to induction chemoradiation therapy in patients with locally advanced rectal adenocarcinoma. Ann Surg Oncol 5: 203-208, 1998.

32. Spitz FR, Giacco GG, Hess K, Larry L, Rich TA, Janjan N, Cleary KR and Skibber JM: p53 immunohistochemical staining predicts residual disease after chemoradiation in patients with high-risk rectal cancer. Clin Cancer Res 3: 1685-1690, 1997.

33. Esposito G, Pucciarelli S, Alaggio R, Giacomelli L, Marchiori E, Iaderosa GA, Friso ML, Toppan P, Chieco-Bianchi L and Lise M: P27kip1 expression is associated with tumor response to preoperative chemoradiotherapy in rectal cancer. Ann Surg Oncol 8: 311-318, 2001.

34. Lin LC, Lee HH, Hwang WS, Li CF, Huang CT, Que J, Lin KL, Lin FC and Lu CL: p53 and p27 as predictors of clinical outcome for rectal-cancer patients receiving neoadjuvant therapy. Surg Oncol 15: 211-216, 2006.

35. Spindler KL, Nielsen JN, Lindebjerg J and Jakobsen A: Germline polymorphisms may act as predictors of response to preoperative chemoradiation in locally advanced T3 rectal tumors. Dis Colon Rectum 50: 1363-1369, 2007

36. Giralt J, de las Heras M, Cerezo L, Eraso A, Hermosilla E, Velez D, Lujan J, Espin E, Rosello J, Majó J, et al: The expression of epidermal growth factor receptor results in a worse prognosis for patients with rectal cancer treated with preoperative radiotherapy: A multicenter, retrospective analysis. Radiother Oncol 74: 101-108, 2005.

37. Kim JS, Kim JM, Li S, Yoon WH, Song KS, Kim KH, Yeo SG, Nam JS and Cho MJ: Epidermal growth factor receptor as a predictor of tumor downstaging in locally advanced rectal cancer patients treated with preoperative chemoradiotherapy. Int J Radiat Oncol Biol Phys 66: 195-200, 2006.

38. Jakob C, Liersch T, Meyer W, Becker H, Baretton GB and Aust DE: Predictive value of Ki67 and p53 in locally advanced rectal cancer: Correlation with thymidylate synthase and histopathological tumor regression after neoadjuvant 5-FU-based chemoradiotherapy. World J Gastroenterol 14: 1060-1066, 2008

39. Kim NK, Park JK, Lee KY, Yang WI, Yun SH, Sung J and Min JS: p53, BCL-2 and Ki-67 expression according to tumor response after concurrent chemoradiotherapy for advanced rectal cancer. Ann Surg Oncol 8: 418-424, 2001

40. Kudrimoti M, Lee EY, Kang Y, Ahmed M and Mohiuddin M: Genetic markers predictive of response to induction chemoradiotherapy for locally advanced rectal cancers. J Ky Med Assoc 105: 18-22, 2007.

41. Chang HJ, Jung KH, Kim DY, Jeong SY, Choi HS, Kim YH, Sohn DK, Yoo BC, et al: Bax, a predictive marker for therapeutic response to preoperative chemoradiotherapy in patients with rectal carcinoma. Hum Pathol 36: 364-371, 2005. 